\title{
Forearm vasodilator responses to environmental stress and reactive hyperaemia are impaired in young South Asian men
}

\author{
Natalie G. Ormshaw ${ }^{1} \cdot$ Rehan T. Junejo $^{1}$ - Janice M. Marshall ${ }^{1}$
}

Received: 23 April 2017 / Accepted: 19 February 2018 / Published online: 3 March 2018

(c) The Author(s) 2018. This article is an open access publication

\begin{abstract}
Purpose Prevalence of cardiovascular disease (CVD) is greater in South Asians (SAs) than White Europeans (WEs). Endothelial dysfunction and blunted forearm vasodilatation to environmental stressors have been implicated in CVD. We investigated whether these features are present in young SA men.

Methods In 15 SA and 16 WE men (19-23 years), we compared changes in forearm blood flow, arterial blood pressure (ABP), forearm vascular conductance (FVC), heart rate, and electrodermal resistance (EDR; sweating) following release of arterial occlusion (reactive hyperaemia endothelium-dependent) and 5 single sounds at 5-10 min intervals (stressors).

Results All were normotensive. Peak reactive hyperaemia was smaller in SAs than WEs (FVC increase: $0.36 \pm 0.038$ vs $0.44 \pm 0.038$ units; $P<0.05$ ). Furthermore, in WEs, mean FVC increased at 5, 15, and $20 \mathrm{~s}$ of each sound (vasodilatation), but increased at $5 \mathrm{~s}$ only in SAs, decreasing by $20 \mathrm{~s}$ (vasoconstriction). This reflected a smaller proportion of SAs showing forearm vasodilatation at $15 \mathrm{~s}(5 / 15 \mathrm{SAs}$ vs $11 / 16$ WEs: $P<0.01)$, the remainder showing vasoconstriction. Concomitantly, WEs showed greater bradycardia and EDR changes. Intra-class correlation analyses showed that all responses were highly reproducible over five sounds in both WEs and SAs. Moreover, sound-evoked changes in ABP and FVC were negatively correlated in each ethnicity $(P<0.01)$. However, WEs showed preponderance of forearm vasodilatation and depressor responses; SAs showed preponderance of vasoconstriction and pressor responses.

Conclusions Endothelium-dependent vasodilatation is blunted in young SA men. This could explain their impaired forearm vasodilatation and greater pressor responses to repeated environmental stressors, so predisposing SAs to hypertension and CVD.
\end{abstract}

Keywords Reactive hyperaemia $\cdot$ Forearm dilatation $\cdot$ Mental stress $\cdot$ South Asian

$\begin{array}{ll}\text { Abbreviations } \\ \text { ABP } & \text { Arterial blood pressure } \\ \text { ANOVA } & \text { Analysis of variance } \\ \text { CVD } & \text { Cardiovascular disease } \\ \text { EDR } & \text { Electrodermal response } \\ \text { FBF } & \text { Forearm blood flow } \\ \text { FMD } & \text { Flow-mediated dilatation } \\ \text { FVC } & \text { Forearm vascular conductance } \\ \text { HR } & \text { Heart rate } \\ \text { ICC } & \text { Intra-class correlation }\end{array}$

Communicated by Carsten Lundby.

Janice M. Marshall

j.m.marshall@bham.ac.uk

1 Institute of Cardiovascular Science, College of Medical and Dental Sciences, The Medical School, University of Birmingham, Birmingham B15 2TT, UK

$\begin{array}{ll}\text { MSNA } & \text { Muscle sympathetic nerve activity } \\ \text { NO } & \text { Nitric oxide } \\ \text { NOS } & \text { Nitric oxide synthase } \\ \text { PGs } & \text { Prostaglandins } \\ \text { PSS } & \text { Perceived Stress Scale } \\ \text { S } & \text { Sound } \\ \text { SA } & \text { South Asian } \\ \text { WE } & \text { White European }\end{array}$

\section{Introduction}

Population studies in the UK, Canada and USA indicate that South Asians (SAs) with ethnic roots in India, Pakistan, Bangladesh, Sri Lanka, or Nepal, have a 2-3-fold greater risk of death from all cardiovascular disease (CVD) than those of White European origin (WE; Gupta et al. 2006; Rana et al. 2014; Wild et al. 2007). Moreover, SAs present 
at younger age with coronary artery disease and heart failure and have higher prevalence of hypertension, the common forerunner of CVD (Agyemand and Bhopal 2006; Gupta et al. 2006). The prevalence of type 2 diabetes is also higher in SAs than WE (Gupta et al. 2006). However, SA men and women (20-60 years) in the USA, who were non-diabetic and normotensive had higher plasma insulin and showed blunted brachial artery vasodilatation to insulin relative to age-matched WEs (Raji et al. 2004). Furthermore, non-diabetic, normotensive SA men (20-40 years) resident in the UK showed higher plasma insulin, smaller flow-mediated dilatation (FMD) and smaller tonic dilator influence of nitric oxide (NO) than WEs (Murphy et al. 2007). These findings suggest that endothelial dilator function deteriorates at an earlier age in SA than WE men and may precede development of hypertension and diabetes.

It is widely accepted that psychosocial stress is an important "modifiable" risk factor for CVD as identified in the INTERHEART study of acute myocardial infarction in 52 countries (Yusuf et al. 2004). Consistent with this, metaanalysis of longitudinal studies of cardiovascular responses to laboratory mental stress and future cardiovascular status demonstrated that greater pressor reactivity to mental stress in young individuals predicted future development of hypertension (Chida and Steptoe 2010). Whether enhanced pressor responses to mental stress and hypertension are causally linked is controversial (Fonkoue and Carter 2015; Lambert and Schlaich 2004; Oparil et al. 2003). However, in normotensives, a single episode of mental stress attenuated endothelium-dependent dilatation in forearm for at least $4 \mathrm{~h}$ (Ghiadoni et al. 2002). Furthermore, the pattern of vascular response evoked by acute mental stress includes muscle vasodilatation (Brod et al. 1959; Halliwill et al. 1997; Hilton 1982) and this vasodilatation is labile in a way that seems likely to facilitate development of hypertension in some individuals. Thus, muscle vasodilator responses evoked by stress tests habituated on repetition and reversed to vasoconstriction in some individuals (Edwards et al. 1998, 1999; Zbrozyna and Westwood 1988). Furthermore, a decrease in muscle sympathetic nerve activity (MSNA) occurred in $\sim 50 \%$ of individuals and habituated on repetition of the stress stimulus. Recurrent increases in MSNA occurred in the remainder and they showed larger and more persistent increases in arterial blood pressure (Donadio et al. 2002, 2012). Similarly, young borderline hypertensives with hypertensive parents showed greater pressor responses to mental stress and smaller decreases in MSNA than young normotensive men with normotensive parents (Matsukawa et al. 1991; Fonkoue et al. 2016). Moreover, mental stress evoked smaller forearm vasodilator responses in young borderline hypertensives than normotensives, while muscle vasodilator responses habituated in young normotensives with hypertensive parents and in borderline hypertensives
(Schneider et al. 2003; Schwartz et al. 2011; Zbrozyna and Krebbel 1985).

In view of these findings, we hypothesised that the forearm vasodilator response to a recognised sound stress stimulus (see Edwards et al. 1998, 1999) would be smaller and habituate more readily on repetition in young SAs than young WEs, while other components would be larger. We also hypothesised that endothelium-dependent, reactive hyperaemia in forearm would be smaller in SAs than WEs. A brief account of our results has been published in abstract form (Ormshaw et al. 2016).

\section{Methods}

The study was performed on 31 male subjects aged $20.80 \pm 0.16$ years (mean \pm SEM, range 19-23) of either South Asian (SA, $n=15$ ), or White European (WE, $n=16$ ) ethnicity. Ethnicity was self-reported (Office for National Statistics 2018); all were UK residents with both parents of the same ethnicity. One subject in each ethnic group had type 1 diabetes; otherwise, they had no known medical condition. They were all non-smokers, except one in each group who had quit smoking for at least 12 months. All experiments were performed in accordance with the Helsinki Declaration and approved by the University of Birmingham Ethics Committee. Subjects were recruited from students of the University of Birmingham, UK and gave written, informed consent.

Each subject attended a preliminary session during which he was familiarized with the recording equipment, completed Cohen's Perceived Stress Scale questionnaire (PSS; Cohen et al. 1983) and a questionnaire dealing with personal characteristics. We used a semi-quantitative scoring system based on the UK Diabetes and Diet Questionnaire (UKDDQ) for fresh fruit and vegetable consumption, as an index of anti-oxidant vitamin $\mathrm{C}$ intake. Alcohol consumption was scored as units/week. Level of elective physical activity was assessed according to the International Physical Activity Questionnaire short version, allowing subjects to indicate time spent in moderate and vigorous exercise per week and scored as MET min/week (The IPAQ Group 2015). Time spent in dynamic exercise (e.g., running, jogging) vs resistance exercise (e.g., press-ups, dumbbell lift) was calculated as percentage of total time. In addition, measurements of resting arterial blood pressure (ABP) were taken with a sphygmomanometer.

\section{Experimental protocol}

Subjects were asked to refrain from consuming alcohol, a heavy meal and undertaking vigorous exercise for at least $24 \mathrm{~h}$ prior to the experiment. Experiments were performed 
in a quiet room with the subject sitting on a couch, with the backrest inclined at $45^{\circ}$ and facing away from the equipment to avoid distractions. Both arms were supported at heart level. ABP was continuously recorded from the right middle finger using a Finapres (Ohmeda 2300, BOC Health Care). Forearm blood flow (FBF) was recorded by venous occlusion plethysmography (strain gauge and gadolinium-filled silastic tubing; EC6, Hokanson Inc.) following inflation of a cuff around the upper arm cuff to $50 \mathrm{mmHg}$ using a rapid inflation system (AG101 Cuff Inflator Air Source; E20 Rapid Cuff Inflator Hokanson Inc.). Values of FBF were computed off-line as the slope over the first two heartbeats following venous occlusion. A second cuff wrapped around the wrist was inflated to $200 \mathrm{mmHg}$ immediately prior to each recording of FBF to exclude blood flow to the hand. Forearm vascular conductance (FVC) was calculated as FBF/ mean ABP. Electrodermal response (EDR) was recorded as an index of sweating, via an Amplifier (GSR Amp FE116; AD Instruments), by electrodes (MLT116; AD Instruments) attached to the first and third finger of the right hand, as maximum change from the baseline. Mean ABP and heart rate (HR) generated from the Finapres recordings, FBF and EDR were recorded to a computer via MacLab software (PowerLab/85P, AD instruments).

\section{Reactive hyperaemia}

After a stabilization period of $15 \mathrm{~min}$, four baseline measurements of FBF were taken at $30 \mathrm{~s}$ intervals. The upper arm cuff was then inflated to $200 \mathrm{mmHg}$ for $2 \mathrm{~min}$. At $2 \mathrm{~min}$, the upper arm cuff was rapidly deflated: FBF was measured immediately and at $15,30,60,90$, and 120 s post-occlusion.

\section{Repeated sounds}

The subject closed his eyes for this part of the protocol to prevent anticipatory stress that might have been triggered by the subject seeing the experimenter initiate the sound stimulus. Five consecutive sounds (S1-S5: $100 \mathrm{~dB}, 1.5 \mathrm{kHz}$; Edwards et al. 1998) each of $30 \mathrm{~s}$ duration were delivered through headphones by an audiometer (Maico MA40, Graystad, Medical), at randomized intervals of 5-10 min. FBF was recorded at 5,15 , and $20 \mathrm{~s}$ into each sound: $5 \mathrm{~s}$ was the earliest timepoint at which we could measure and gave us an index of the very early part of the response when ABP is rising; $15 \mathrm{~s}$ is the timepoint we have used before when $\mathrm{ABP}$ and skin vasoconstriction have reached their peak (Edwards et al. 1998); a measurement at $20 \mathrm{~s}$ was included to indicate whether the forearm vascular response stabilised. The subject rated each sound on a scale of $0-10,10$ being the most stressful.

\section{Statistical analyses}

Results are expressed as mean \pm SEM. Baseline comparisons between ethnicities were made with Student's unpaired $t$ test. Proportions of subjects with hypertensive vs normotensive parents, those born in UK vs non-UK and time spent in dynamic vs resistance exercise were compared by Fisher's exact test. For reactive hyperaemia, within and between group analyses were performed by two-way repeated measures ANOVA. Responses evoked by sound were compared by mixed model ANOVA with repeated measures. Post-hoc comparisons were made with Tukey's HSD for multivariate comparisons. Individuals were divided into "vasodilators" and "vasoconstrictors" according to the direction of change in FVC at $15 \mathrm{~s}$ into $\mathrm{S} 1$ when the evoked change in ABP and cutaneous vasoconstriction reach their maximum (Edwards et al. 1998). The proportions of vasodilators and vasoconstrictors amongst WEs and SAs were compared by Fisher's exact test. The consistency of changes evoked in each variable at $15 \mathrm{~s}$ into the five sounds was tested by intra-class correlation (ICC) analysis; the coefficient of consistency [Cronbach's alpha $(\alpha)$ value] is reported with two-tailed $P$ values. Relationships between variables within individuals of each ethnicity were investigated by linear regression analysis using Pearson's correlation coefficient $(r)$ and coefficient of determination $\left(r^{2}\right) . P<0.05$ was taken to indicate statistical significance.

\section{Results}

\section{Baseline characteristics}

There were no significant differences between the ethnic groups for anthropometric data and lifestyle variables except caffeine intake, which was greater in WEs (Table 1); it should be noted that all subjects refrained from caffeine intake for at least $24 \mathrm{~h}$ before the experiment. There were also no differences between ethnic groups for resting $\mathrm{ABP}$, or cardiovascular baselines recorded at the beginning of the experimental protocol (Table 2).

\section{Reactive hyperaemia}

In SAs and WEs, FVC was increased at time 0 and returned to baseline by $2 \mathrm{~min}$ following release of occlusion $(P<0.01$ in each case). The increase in FVC was significantly greater in the WEs than SAs at 0 and $15 \mathrm{~s}$ ( $P=0.012 ; 0.014$, respectively, Fig. 1). 
Table 1 Characteristics of South Asians and White Europeans

\begin{tabular}{llll}
\hline & South Asians $(n=15)$ & White Europeans $(n=16)$ & $P$ value \\
\hline Age (years) & $20.6 \pm 0.214$ & $21.1 \pm 0.232$ & 0.155 \\
BMI $\left(\mathrm{kg} / \mathrm{m}^{2}\right)$ & $22.5 \pm 0.754$ & $21.9 \pm 0.578$ & 0.529 \\
Exercise (MET min/week) & $2702.2 \pm 435.526$ & $2094 \pm 278.779$ & 0.257 \\
Dynamic: resistive exercise (\%) & $74: 26$ & $79: 21$ & 0.094 \\
Vit C-containing portions/day & $5.87 \pm 0.274$ & $6.69 \pm 0.463$ & 0.117 \\
Caffeine intake (cups/day) & $0.533 \pm 0.236$ & $1.81 \pm 0.410$ & 0.003 \\
Parental hypertension (Y/N) & $4 / 11$ & $3 / 13$ & 0.291 \\
UK birth (Y/N) & $12 / 3$ & $15 / 1$ & 0.239 \\
SBP (mmHg) & $124 \pm 2.55$ & $122 \pm 1.78$ & 0.578 \\
DBP (mmHg) & $74.6 \pm 1.76$ & $73.0 \pm 1.73$ & 0.536 \\
MABP (mmHg) & $90.8 \pm 1.93$ & $89.8 \pm 1.55$ & 0.399 \\
HR (beats/min) & $78.2 \pm 2.88$ & $70.7 \pm 3.19$ & 0.0843 \\
\hline
\end{tabular}

All values are mean \pm SEM unless otherwise stated. $P$ value: WEs vs SAs. For further description of scoring systems see text

$B M I$ body mass index, $H R$ heart rate, $S B P$ systolic blood pressure, $D B P$ diastolic blood pressure, $M A B P$ mean arterial blood pressure, exercise elective physical activity scored as MET min/week spent in moderate exercise and vigorous exercise, Vit C-containing portions high vitamin C-containing fruit and vegetable portions
Table 2 Baseline values recorded in South Asians and White Europeans

\begin{tabular}{lccc}
\hline & South Asians & White Europeans & $P$ value \\
\hline MABP (mmHg) & $82.1 \pm 3.4$ & $88.8 \pm 3.5$ & 0.184 \\
HR (beats/min) & $66.5 \pm 2.3$ & $67.8 \pm 2.4$ & 0.703 \\
FBF $(\mathrm{ml} \mathrm{100/ml/min)}$ & $6.90 \pm 0.724$ & $6.16 \pm 0.661$ & 0.461 \\
FVC $(\mathrm{CU})$ & $0.088 \pm 0.012$ & $0.069 \pm 0.007$ & 0.166 \\
\hline
\end{tabular}

$P$ value: WEs vs SAs

$M A B P$ mean arterial blood pressure, $H R$ heart rate, $F B F$ forearm blood flow, $F V C$ forearm vascular conductance, $C U$ conductance units

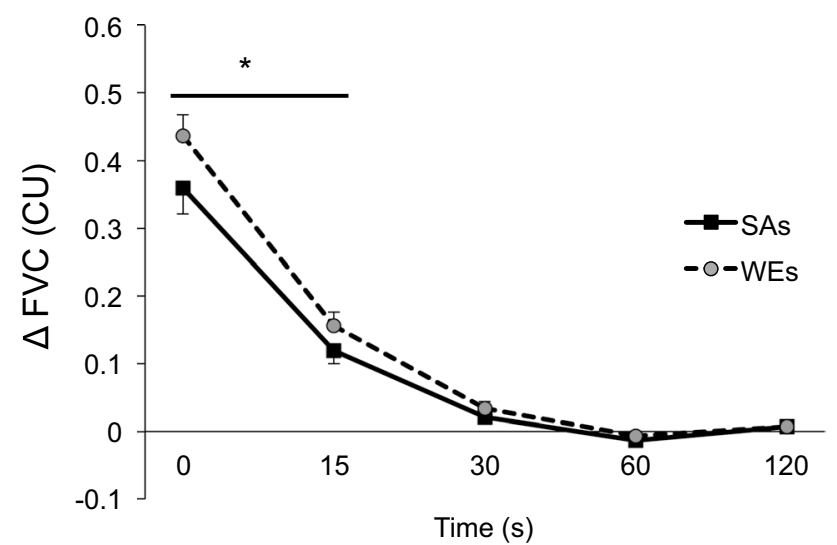

Fig. 1 Reactive hyperaemia evoked in forearm of White Europeans (WEs) and South Asians (SAs). Solid black symbols: SAs. Grey filled symbols: WEs. $* P<0.05$ WEs vs SAs

\section{Responses evoked by sound}

Considering group mean values (Fig. 2), the variability was high in both groups, but the pattern of response evoked by each sound stimulus (S1-S5) differed significantly between SAs and WEs $(P<0.05$ for all variables except ABP). Thus, WEs showed a mean increase in $\mathrm{FVC}$ at 5,15 , and $20 \mathrm{~s}$ into each sound, whereas SAs showed a mean increase in FVC at $5 \mathrm{~s}$, with a decrease in FVC by $20 \mathrm{~s}(P=0.007$ for WE vs SA across S1-S5; $P<0.05$ for each sound). There was no significant difference between WE and SA for change in ABP across S1-S5 $(P=0.74)$, but WEs showed a greater decrease in HR across $\mathrm{S} 1-\mathrm{S} 5(P=0.01 ; P<0.05$ for each sound). EDR was consistently greater in WEs than SAs ( $P=0.01$ across $\mathrm{S} 1-\mathrm{S} 5 ; P<0.05$ for each sound).

Despite the group variability, changes evoked by S1-S5 within individuals were reproducible. The majority of WEs and SAs showed an increase in FVC at $5 \mathrm{~s}$ (11/16 and 11/15, respectively). However, by $15 \mathrm{~s}$ of $\mathrm{S} 1$, a greater proportion of WEs than SEs showed an increase in FVC (dilatation; 11/16 vs $5 / 15$, respectively, Fisher's exact test: $P=0.006$ ). Correspondingly, a greater proportion of SAs showed a decrease in FVC (constriction; 10/15 vs 5/16). ICC analysis revealed that the reproducibility of responses at $15 \mathrm{~s}$ was high in individuals amongst both WEs and SAs: Cronbach's $\alpha$ for WEs and SAs, respectively, were 0.94 and 0.80 for FVC, 0.94 and 0.86 for $\mathrm{ABP}, 0.87$ and 0.88 for HR, and 0.98 and 0.92 for EDR $(P<0.001$ in each case). In other words, there was no obvious habituation of forearm vascular response with repetition of sounds in either WEs or SAs; within group 


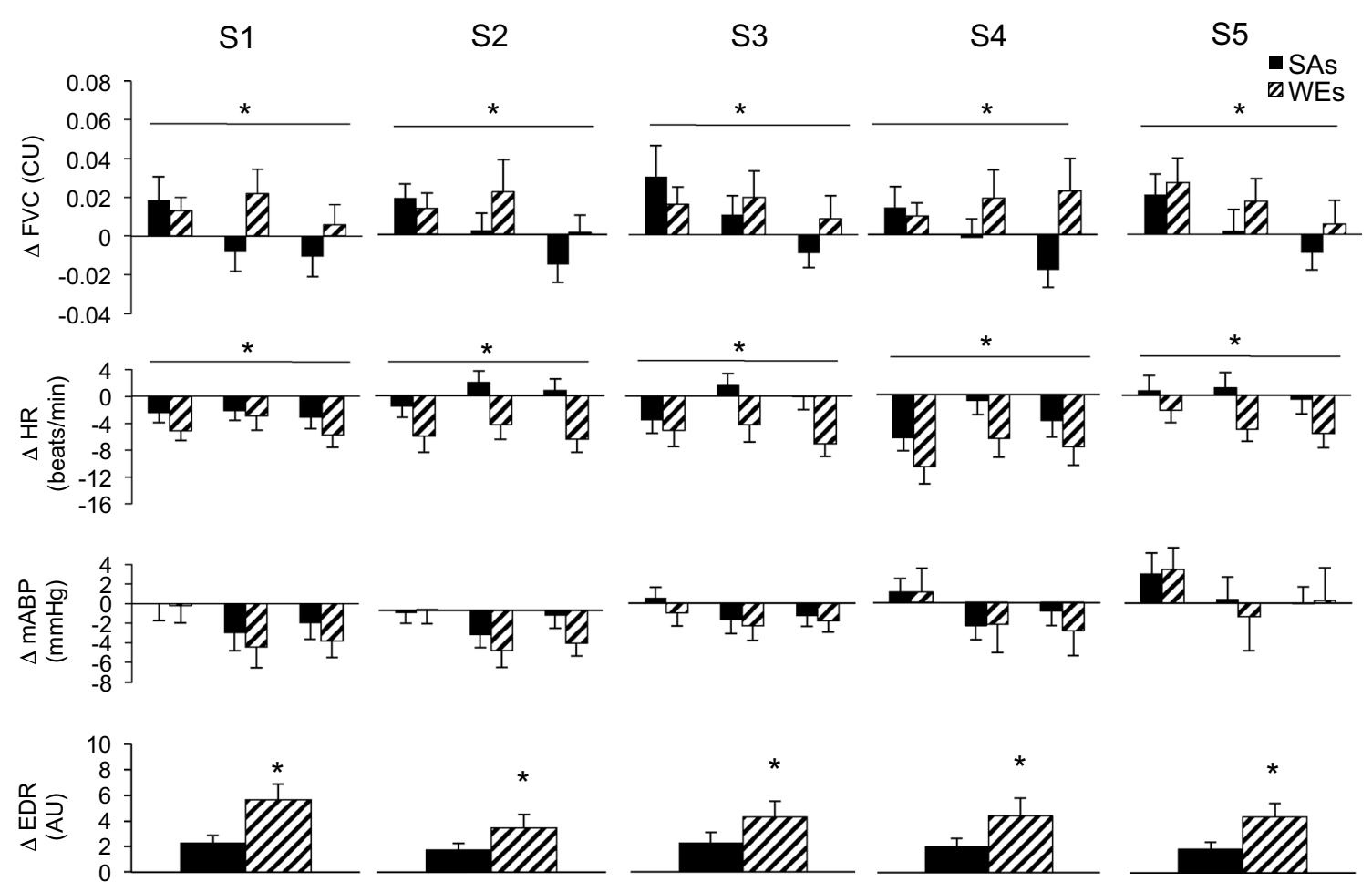

Fig. 2 Cardiovascular and sweating responses evoked in response to sounds 1-5 in South Asians (SAs) and White Europeans (WEs). Cardiovascular variables were measured at 5,15 , and $20 \mathrm{~s}$ into each

comparisons for effect of sound repetition on change in FVC; $P=0.975$ and $P=0.938$, respectively. In fact, only two WEs who showed an increase in FVC at S1, switched to a substantial decrease in FVC by S4 and S5, consistent with habituation of forearm vasodilatation (Edwards et al. 1998, 1999; Zbrozyna and Westwood 1988); no vasodilator SAs showed such behaviour.

When WEs and SAs were categorized as "vasodilators" or "vasoconstrictors" by the direction of change in FVC at $15 \mathrm{~s}$, baselines were not significantly different between the subgroups apart from HR, which was lower in vasoconstrictors (Table 3). However, the pattern of response evoked by S1-S5 was different between vasodilators and sound, whereas sweating (EDR) was averaged over $30 \mathrm{~s}$ (for further details, see text). Solid black columns: SAs. Cross-hatched columns: WEs. *WE vs SA, $P<0.05$

vasoconstrictors (see Fig. 3, for S1); data for S2-S5 are not shown, but consistent with the ICC values, and these responses were highly comparable to those of $\mathrm{S} 1$. Changes in FVC between the vasodilator and vasoconstrictor subgroups were not compared, because we chose to separate individuals into subgroups on this basis. However, the changes in ABP and EDR differed between vasodilators and constrictors when WEs and SAs were considered together $(P<0.001$ and $P=0.045$, respectively, across S1-S5). Mean ABP fell by $\sim 5-10 \mathrm{mmHg}$ at $15 \mathrm{~s}$ in vasodilators (Fig. 3), but showed a trend to increase by up to $5 \mathrm{mmHg}$ in vasoconstrictors (Fig. 3). EDR responses were greater in vasodilators; any apparent trend for EDR responses to be greater in WEs than

Table 3 Baseline values in South Asian and White European constrictor and dilator subgroups

\begin{tabular}{lcccccc}
\hline & \multicolumn{2}{l}{ South Asians } & & & White Europeans & $P$ value \\
\cline { 2 - 3 } & Dilators $(n=5)$ & Constrictors $(n=10)$ & & Dilators $(n=11)$ & Constrictors $(n=5)$ \\
\hline MABP (mmHg) & $81.0 \pm 4.61$ & $82.7 \pm 4.75$ & & $88.4 \pm 4.29$ & $83.6 \pm 3.37$ \\
HR (beats/min) & $69.6 \pm 4.95$ & $65.0 \pm 2.57$ & & $71.3 \pm 2.68$ & $60.2 \pm 2.79$ \\
FBF (ml 100/ml/min) & $6.08 \pm 0.951$ & $7.31 \pm 0.982$ & & $5.90 \pm 0.837$ & $6.74 \pm 1.13$ & 0.330 \\
FVC (CU) & $0.076 \pm 0.014$ & $0.094 \pm 0.017$ & & $0.063 \pm 0.007$ & $0.082 \pm 0.015$ \\
\hline
\end{tabular}

$P$ value: dilators vs constrictors

$M A B P$ mean arterial blood pressure, $H R$ heart rate, $F B F$ forearm blood flow, $F V C$ forearm vascular conductance, $C U$ conductance units 

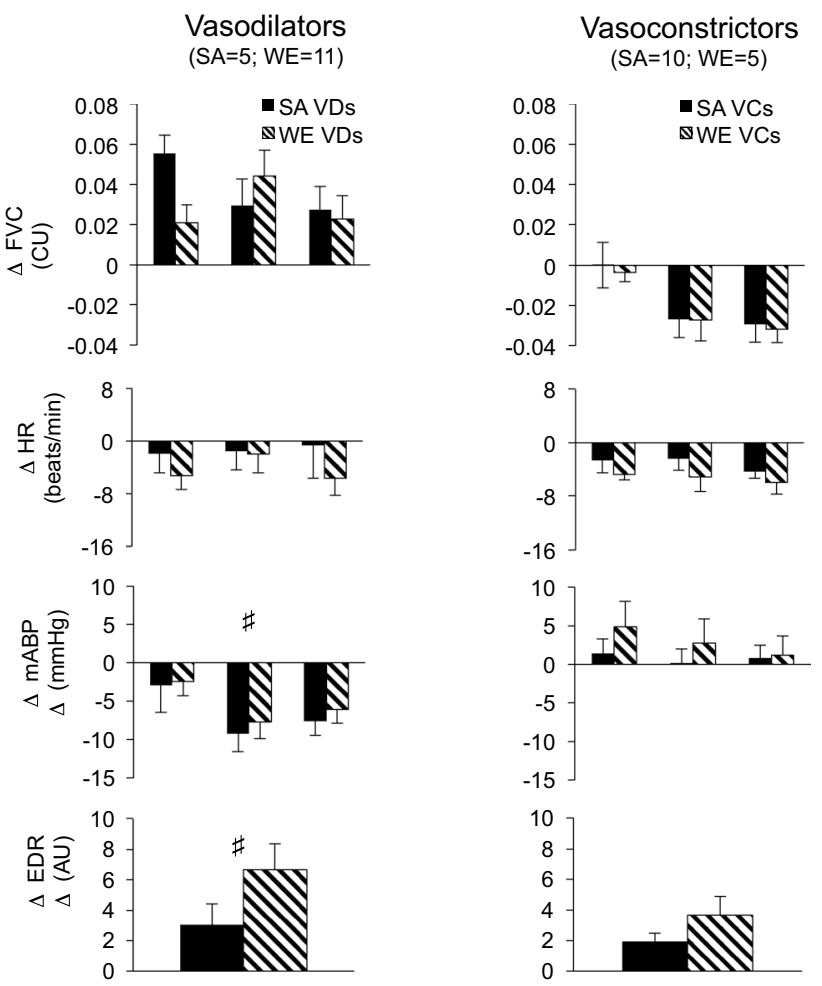

Fig. 3 Cardiovascular and sweating responses evoked in response to sound 1 in vasodilator and vasoconstrictor SAs and WEs (left, right, respectively). Solid black columns: SAs. Cross-hatched columns: WEs. Abbreviations and timing of measurements as in Fig. 2. ${ }^{\#} P<0.05$ for changes evoked by $\mathrm{S} 1$ in vasodilators vs changes evoked by $\mathrm{S} 1$ in vasoconstrictors

SAs was not statistically significantly $(P=0.197,0.153$ for vasodilators, vasoconstrictors, respectively).

Across S1-S5, the preponderance of repeated forearm vasodilator responses and falls in ABP amongst WEs, but repeated forearm vasoconstrictor responses and rises in $\mathrm{ABP}$ amongst SAs was also evident when change in
$\mathrm{ABP}$ was plotted against change in $\mathrm{FVC}$ for each sound in each individual (Fig. 4). In both WEs and SAs, changes in $\mathrm{ABP}$ at $15 \mathrm{~s}$ were negatively correlated with changes in FVC $\left(r=0.70, r^{2}=0.49 ; P<0.001\right.$ and $r=0.43, r^{2}=0.18$; $P=0.001$, respectively).

\section{Sound stress rating and perceived stress score}

Sound stress ratings were greater in WEs than SAs across S1-S5 ( $P=0.039$; mean sound stress rating across S1-S5: $6.19 \pm 0.17$ vs $5.76 \pm 0.19$, respectively). The PSS showed a trend to be greater in WEs than SAs $(12.5 \pm 1.74$ vs $8.73 \pm 1.17$, respectively; $P=0.101$ ). There was no correlation between SS score and change in FVC at $15 \mathrm{~s}$ in WEs or SAs (WEs: $r=0.13, r^{2}=0.02 ; P=0.26$; SAs: $r=0.19$, $\left.r^{2}=0.03 ; P=0.13\right)$.

\section{Discussion}

The new findings of the present study are first that young SA men showed blunted reactive hyperaemia in forearm relative to young WE men. Second, repeated sound stimuli evoked repeatable, maintained forearm vasodilator response in the majority of WEs, whereas most SAs consistently showed transient forearm vasodilatation, which waned to vasoconstriction.

These disparities are noteworthy, because both the SAs and WEs were from a young age range (19-23 years) with no obvious signs of hypertension or other CVD. They had BMI values below those considered a risk for type 2 diabetes and CVD (23 and $25 \mathrm{~kg} / \mathrm{m}^{2}$, respectively; NICE Guidance 2014). They were also recreationally active, with alcohol consumption well below the recommended limit. The great majority were born in the UK and similar small proportions had hypertensive parents (4/15 SAs; 3/16 WEs). Thus, the
Fig. 4 Relationship between evoked change in FVC and ABP in response to each of sounds S1-S5 in individual SAs (left) and WEs (right). In each ethnicity, subjects were categorized as vasoconstrictors (VCs) or vasodilators (VDs) by direction of change in FVC at $15 \mathrm{~s}$ into $\mathrm{S} 1$

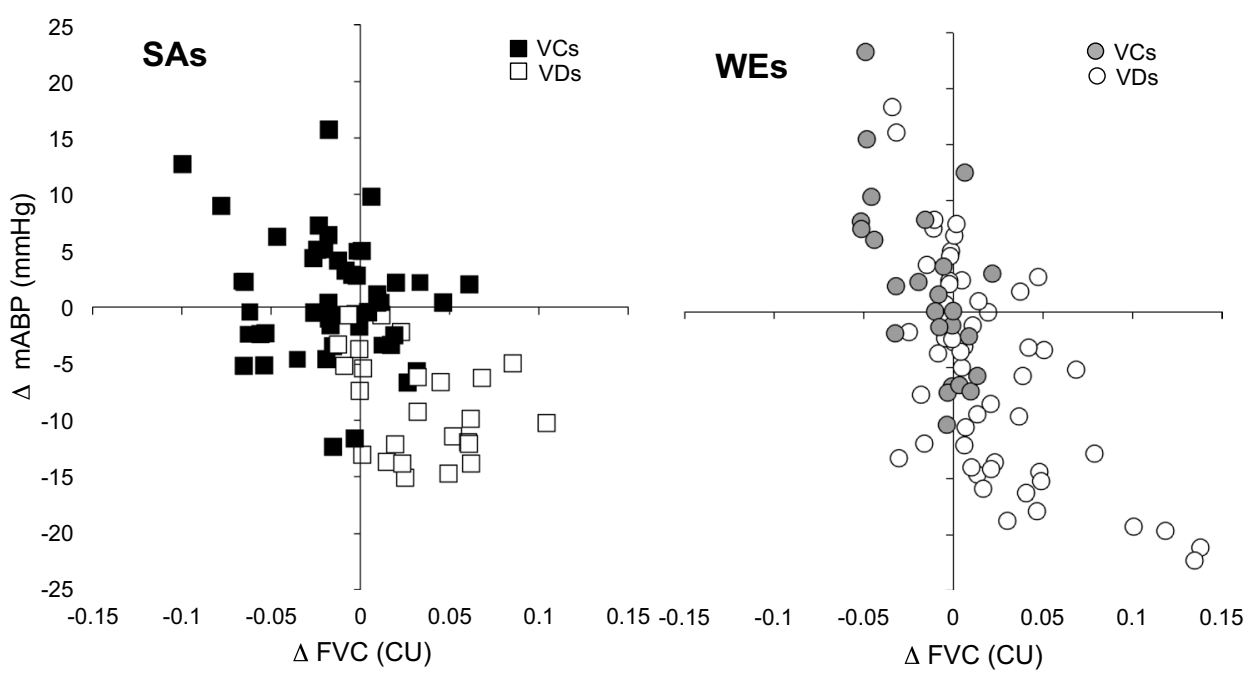


only clear difference between these young SA and WEs was their ethnicity.

\section{Reactive hyperaemia}

The blunted reactive hyperaemia in young SA men relative to WE men is fully consistent with our hypothesis. Reactive hyperaemia is attributed to myogenic dilatation, augmented, and maintained by endothelium-dependent vasodilators generated by hypoxia and shear stress (Carlsson et al. 1987; Engelke et al. 1996). It has been reported that NO contributes to peak reactive hyperaemia in normal subjects, but its contribution is attenuated in patients at high risk of CVD (Dakak et al. 1998). Other deduced prostaglandins (PGs), adenosine, and vascular hyperpolarization contribute to the peak, while NO helps maintain reactive hyperaemia (Carlsson et al. 1987; Engelke et al. 1996; Crecelius et al. 2013; Tagawa et al. 1994). Collectively, these findings are consistent with adenosine, PGs and NO acting interdependently (Carlsson et al. 1987; Ray et al. 2002). The tonic vasodilator influence of NO in forearm was attenuated in SAs aged 20-40 years relative to WEs (Murphy et al. 2007). Thus, it seems likely blunted reactive hyperaemia in young SA men reflects blunted contributions of NO, adenosine and/or PGs. Clearly, these proposals should be investigated.

Interestingly, in a longitudinal study of fit, healthy men in the USA, peak reactive hyperaemia in forearm was shown to be a better prognostic indicator of future CVD than FMD of the brachial artery (Anderson et al. 2011). FMD was not depressed in SAs aged 20-40 years (Murphy et al. 2007). Thus, it is reasonable to suggest that the blunted reactive hyperaemia we describe in young SA men may be an early predictor of increased risk of CVD.

\section{Responses evoked by repeated sound}

Vasodilatation in limb muscle is a characteristic component of the behavioural and autonomic pattern of the alerting or defence response to environmental and mental stressors (Brod et al. 1959; Hilton 1982). Accordingly, the group mean data of the present study indicate that young normotensive WE men who were naïve to the sound stimulus, generally showed well-maintained forearm vasodilator responses to repeated sounds with no habituation within session. This compares favorably with our findings on normotensive WE subjects of wider age range (21-70, mean 39 years) who were similarly naïve, but mainly female: the majority showed forearm vasodilatation at $15 \mathrm{~s}$ which habituated over 3 sessions, but not within the first session (Edwards et al. 1998).

On the other hand, judging from group mean data, young SA men generally showed only transient forearm vasodilatation that waned to vasoconstriction with each sound.
This agrees with our hypothesis that young SA men would show blunted forearm vasodilatation relative to WEs. It was reported that normotensive black African Americans aged $\sim 42$ years, showed blunted forearm vasodilatation to mental stress relative to White Americans (Cardillo et al. 1998a). We believe that ours is the first evidence that forearm vasodilatation evoked by environmental stress is blunted in young normotensive SA men relative to WE men.

In our previous study, WEs showed a rise in ABP and tachycardia with each sound (Edwards et al. 1998) as is typical of the alerting response (Brod et al. 1959; Hilton 1982), whereas in the present study, young WE men and SA men showed no net pressor response and a trend for bradycardia. This disparity seems likely to reflect the unpleasantness of the stimulus: a lower frequency $(1.5 \mathrm{kHz})$ in the present study vs $2 \mathrm{kHz}$ (Edwards et al. 1998). Thus, low intensity sounds evoked vagal bradycardia, and higher intensity sounds evoked sympathetic tachycardia (Gianaros and Quigley 2001; Turpin et al. 1999). Variable HR responses were also reported to electric shocks, bradycardia being more common (Donadio et al. 2002).

\section{Limb vascular responses in individuals}

The majority of young WE and SA men showed an increase in FVC (vasodilatation) at $5 \mathrm{~s}$ into each sound, but diverged thereafter. Thus, we divided them into "vasodilators" and "vasoconstrictors" according to the direction of change in FVC at $15 \mathrm{~s}$ when ABP and cutaneous vasoconstriction reach their peak (Edwards et al. 1998). This revealed the proportion of vasodilators was much smaller in SAs than WEs: $\sim$ one-third vs two-thirds, respectively. However, importantly, within each ethnicity, the direction of change in FVC was highly consistent within individuals from the first to the fifth sound; just two vasodilator WEs showed habituation of the vasodilatation within session. As far as we are aware, this is the first evidence that forearm vascular responses to stress stimuli are reproducible on repetition in any ethnic group. However, it is reminiscent of the finding that when normotensive subjects were categorized on the basis of whether their MSNA decreased, or increased in response to an electric shock: 50-60\% showed a decrease in MSNA, some showing habituation on repetition and the remainder showed an increase in MSNA. Furthermore, the direction of change in MSNA was reproducible between electric shock and mental stress and when electric shocks were repeated after several months (Donadio et al. 2002, 2012).

The question arises as to why more SAs than WEs were vasoconstrictors? The EDR (sweating) response and stress ratings for the sound stimuli were lower in SAs than WEs and SAs tended to report lower perceived stress scores for everyday life. Thus, the dominance of vasoconstrictors amongst SAs was not associated with greater responsiveness 
to, or perception of, mental stress. This was worth considering because some, but not all studies showed a positive correlation between intensity of perceived stress and increase in MSNA (Callister et al. 1992; Carter and Ray 2009; Donadio et al. 2012). In addition, the higher proportion of vasoconstrictors in SAs cannot be explained by family history of hypertension (Matsukawa et al. 1991) for a similar, small number SAs and WEs had hypertensive parents.

It seems more likely the disparity reflects the mechanisms that underlie the forearm vascular response. In many individuals, a sudden electric shock evoked a decrease in MSNA within one cardiac cycle (Donadio et al. 2002). Thus, a decrease in MSNA may well have contributed to the forearm vasodilatation that occurred at $5 \mathrm{~s}$ into the sound in the majority of WEs and SAs. Maintained forearm vasodilatation evoked by mental stress lasting $5 \mathrm{~min}$ has been reported to be accompanied by a long-lasting decrease in forearm MSNA (Halliwill et al. 1997), or by a progressive increase in forearm MSNA (Carter et al. 2005). Thus, it could be a higher proportion of SAs than WEs switched from a decrease in MSNA at $5 \mathrm{~s}$, to an increase in MSNA by $15 \mathrm{~s}$, so explaining the forearm vasoconstriction at 15 and $20 \mathrm{~s}$. This seems unlikely given evidence that changes in MSNA evoked by short and longer-lasting stressors are consistent within individuals (Donadio et al. 2002, 2012).

An alternative explanation is that the disparity reflects a blunted contribution of endothelium-dependent dilatation to the forearm vascular response in SAs. The forearm vasodilatation of mental stress is highly dependent on shear stress-induced release of $\mathrm{NO}$ and the $\beta_{2}$-adrenoceptor effect of adrenaline, which is NO-dependent (Dietz et al. 1994; Halliwill et al. 1997; Seddon et al. 2008). The NO-dependent component of stress-induced forearm vasodilatation was impaired in those with hypertensive parents, in hypertensives and in black African Americans (Cardillo et al. 1998a, b; Schlach et al. 2002; Khan et al. 2015). Thus, it is reasonable to propose that the majority of young SA men showed forearm vasoconstriction because their NO-dependent, and $\beta_{2}$-adrenoceptor mediated dilatation was blunted. The few vasodilator SAs may have been those who showed greater stress-induced release of adrenaline and consequent NOS activation. This agrees with our finding that the vasodilators of both ethnicities showed larger sweating responses than the vasoconstrictors.

\section{Relationships between forearm vascular responses and $A B P$}

In both WEs and SAs, sound-evoked increases in FVC in vasodilators were associated with repeated falls in ABP. This suggests that in these individuals, vasodilatation occurred in both forearms and possibly leg muscles and made a major contribution to the fall in $\mathrm{ABP}$, for any change in $\mathrm{HR}$ was small and the alerting response includes generalized vasoconstriction except in muscle (Brod et al. 1959; Hilton 1982). On the other hand, the trend for ABP to increase repeatedly in the vasoconstrictor WEs and SAs suggests that they showed repeated vasoconstrictor responses in forearms and legs. Similarly, those in whom MSNA decreased in response to short and longer-lasting stressors showed a fall, or smaller rise in ABP than those in whom MSNA increased (Donadio et al. 2002, 2012). Indeed, the negative relationship between sound-evoked changes in ABP and FVC in young WE and SA men is consistent with the positive relationship between stressor-evoked changes in MSNA and ABP (Donadio et al. 2012).

From a wider perspective, the repeatability of FVC responses to stressor stimuli and the dominance of forearm vasoconstrictor responses in SAs indicate that more young SA, than WE men are exposed to the vasoconstrictor, pressor effects of environmental stressors in everyday life. Given the evidence that repeated stress-induced vasoconstriction promotes vascular hypertrophy and development of hypertension (Oparil et al. 2003) and that heightened pressor responsiveness to laboratory stressors is predictive of future CVD (Chida and Steptoe 2010), our findings indicate that young men of SA ethnicity are preferentially exposed to these effects. The presence of high levels of stress in everyday life would be expected to further exacerbate the tendency of SA men to develop hypertension, particularly as acute stress impairs endothelial vasodilator function (Ghiadoni et al. 2002).

\section{Study limitations}

A larger study is required to indicate whether our findings hold for a wider population of young SA men, or encompass young SA women, while longitudinal studies would be required to test whether young vasoconstrictor SAs are indeed at higher risk of hypertension and CVD. Self-reporting of diet and physical activity allowed the study to be noninvasive and facilitated recruitment of subjects. However, blood samples would have allowed assay markers of oxidative stress, lipid profile and insulin resistance, while a standard exercise test would have allowed better comparison of functional capacity. Such tests may have indicated whether young SAs showed higher risk factors for CVD than WEs. Self-reporting may also have led to underestimation of family history of hypertension.

We focused on forearm vasodilator response to repeated sounds as this component of the alerting response is labile and a recognised marker for CVD; recordings of skin blood flow would have indicated whether the vasoconstrictor components also differ between SAs and WEs. Finally, as we used a short, physical stressor, it will be important to 
establish whether our findings can be extrapolated to longer lasting, psychosocial, or cognitive stressors.

\section{Conclusions}

Our novel findings indicate that even in young adulthood, SA men resident in Europe who are physically active with no overt CVD, already show impaired reactive hyperaemia and forearm vasodilator responses to environmental stress relative to matched WE men. We suggest that this largely reflects blunted endothelium-dependent dilatation in young SA men and results in dominant forearm vasoconstrictor responses to stress. Blunted forearm vasodilatation may be an early indication of endothelium dysfunction that increases the risk of SA men developing hypertension and CVD in later life. Moreover, repeated limb vasoconstriction to stressors in everyday life may, over time, exacerbate the pressor response to stress in SA men, facilitating development of hypertension and further CVD.

Acknowledgements The authors would like to thank all the students who volunteered and generously gave their time to take part in this study.

Author contributions JMM conceived the study. RTJ provided much of the laboratory training for NGO and supported her in performing the studies. NGO, RTJ, and JMM each played a part in data extraction, statistical analyses, and interpretation. NGO and JMM played major roles in writing the draft of the MS and all authors contributed to and approved the final MS.

\section{Compliance with ethical standards}

Conflict of interest The authors have no source of funding or conflict of interest to declare.

Open Access This article is distributed under the terms of the Creative Commons Attribution 4.0 International License (http://creativeco mmons.org/licenses/by/4.0/), which permits unrestricted use, distribution, and reproduction in any medium, provided you give appropriate credit to the original author(s) and the source, provide a link to the Creative Commons license, and indicate if changes were made.

\section{References}

Agyemand C, Bhopal RS (2002) Is the blood pressure of South Asian adults in the UK higher or lower than that in European white adults? A review of cross-sectional data. J Hum Hypertens 16:739-751

Anderson TJ, Charbonneau F, Title LM, Buithieu J, Rose MS, Conradson H, Hildebrand K, Fung M. Verma S, Lonn EM (2011) Microvascular function predicts cardiovascular events in primary prevention: long-term results from the Firefighters and Their Endothelium (FATE) study. Circulation 123:163-169

Brod J, Fencl V, Hejl Z, Jirka J (1959) Circulatory changes underlying blood pressure elevation during acute emotional stress (mental arithmetic) in normotensive and hypertensive subjects. Clin Sci 18:269-279

Callister R, Suwarno NO, Seals DR (1992) Sympathetic activity is influenced by task difficulty and stress perception during mental challenge in humans. J Physiol 454:373-387

Cardillo C, Kilcoyne CM, Canon RO III, Panza JA (1998a) Racial differences in the nitric oxide-mediated vasodilator response to mental stress in the forearm circulation. Hypertension 31:1235-1239

Cardillo C, Kilcoyne CM, Cannon RO, Panza JA (1998b) Impairment of the nitric oxide-mediated vasodilator response to mental stress in hypertensive but not in hypercholesterolemic patients. JACC 32:1207-1213

Carlsson I, Sollevi A, Wennmalm A (1987) The role of myogenic relaxation, adenosine and prostaglandins in human forearm reactive hyperaemia. J Physiol 389:147-161

Carter JR, Ray CA (2009) Sympathetic neural responses to mental stress: responders, nonresponders and sex differences. Am J Physiol Heart Circ Physiol 296:H847-853

Carter JR, Kupiers NT, Ray CA (2005) Neurovascular response to mental stress. J Physiol 564:321-327

Chida Y, Steptoe A (2010) Greater cardiovascular responses to laboratory mental stress are associated with poor subsequent cardiovascular risk status: a meta-analysis of prospective evidence. Hypertension 55:1026-1032

Cohen S, Kamarck T, Mermelstein R (1983) A global measure of perceived stress. J Health Soc Behav 24:385-396

Crecelius AR, Richards JC, Luckasen GJ, Larson DG, Dienno FA (2013) Reactive hyperemia occurs via activation of inwardly rectifying potassium channels and $\mathrm{Na}^{+} / \mathrm{K}^{+}$-ATPase in humans. Circ Res 113:1023-1032

Dakak N, Husain S, Mulcahy D, Andrews NP, Panza JA, Waclawiw M, Schenke W, Quyyumi AA (1998) Contribution of nitric oxide to reactive hyperemia impact of endothelial dysfunction. Hypertension 32:9-15

Dietz NM, Rivera JM, Eggener SE, Fix RT, Warner DO, Joyner MJ (1994) Nitric oxide contributes to the rise in forearm blood flow during mental stress in humans. J Physiol 480:361-368

Donadio V, Karlsson T, Elam M, Wallin BG (2002) Interindividual differences in sympathetic and effector responses to arousal in humans. J Physiol 544:293-302

Donadio V, Liguori R, Elam M, Karlsson T, Giannoccaro MP, Pegenius G, Giambattistelli F, Wallin BG (2012) Muscle sympathetic response to arousal predicts neurovascular reactivity during mental stress. J Physiol 590:2885-2896

Edwards CM, Marshall JM, Pugh M (1998) Lack of habituation of the pattern of cardiovascular response evoked by sound in subjects with primary Raynaud's disease. Clin Sci 95:249-260

Edwards CM, Marshall JM, Pugh M (1999) Cardiovascular responses evoked by mild cool stimuli in primary Raynaud's disease: the role of endothelin. Clin Sci 96:577-588

Engelke KA, Halliwill JR, Proctor DN, Dietz NM, Joyner MJ (1996) Contribution of nitric oxide and prostaglandins to reactive hyperemia in the human forearm. J Appl Physiol 81:1807-1814

Fonkoue IT, Carter JR (2015) Sympathetic neural reactivity to mental stress in humans: test-retest reproducibility. Am J Physiol Regul Integr Comp Physiol 309:R1380-R1386

Fonkoue IT, Wang M, Carter JR (2016) Sympathetic neural reactivity to mental stress in offspring of hypertensive parents: 20 years revisited. Am J Physiol Heart Circ Physiol 311:H426-H432

Ghiadoni L, Donald AE, Cropley M, Mullen MJ, Oakley G, Taylor M, O'Connor G, Betteridge J, Klein N, Steptoe A, Deanfield JE (2002) Mental stress induces transient endothelial dysfunction in humans. Circulation 102:2473-2478

Gianaros PJ, Quigley KS (2001) Autonomic origins of a nonsignal stimulus-elicited bradycardia and its habituation in humans. Psychophysiology 38:540-547 
Gupta M, Singh N, Verma S (2006) South Asians and cardiovascular risk: what clinicians should know. Circulation 113:924-929

Halliwill JR, Lawler LA, Eickhoff TJ, Dietz DM, Naus LA, Joyner MJ (1997) Forearm sympathetic withdrawal and vasodilatation during mental stress in humans. J Physiol 504:211-220

Hilton SM (1982) The defence-arousal system and its relevance for circulatory and respiratory control. J Exp Biol 100:159-174

Khan SG, Geer A, Fok HW, Shabeeh H, Brett SE, Shah AM, Chowienczyk PJ (2015) Impaired neuronal nitric oxide synthase-mediated vasodilator responses to mental stress in essential hypertension. Hypertension 65:903-909

Lambert EA, Schlaich MP (2004) Reduced sympathoneural responses to the cold pressor test in individuals with essential hypertension and in those genetically predisposed to hypertension. No support for the "pressor reactor" hypothesis of hypertension development. Am J Hypertens 17:863-868

Matsukawa T, Gotoh E, Uneda S, Miyajima E, Shionoiri H, Tochikubo O, Ishii M (1991) Augmented sympathetic nerve activity in response to stressors in young borderline hypertensive men. Acta Physiol Scand 141:157-165

Murphy C, Kanaganayagam GS, Jiang B, Chowienczyk PJ, Zbinden R, Saha M, Rahman S, Shah AM, Marber MS, Kearney MT (2007) Vascular dysfunction and reduced circulating endothelial progenitor cells in young healthy UK South Asian men. Arterioscler Thromb Vasc Biol 27:936-942

NICE Guidance [LGB13] (2014) Body mass index thresholds for intervening to prevent ill health among black, Asian and other minority ethnic groups. http://publications.nice.org.uk/lgb13. Accessed 2 Mar 2018

Office for National Statistics (2018) Guidance and methodology: ethnic group (online). http://www.ons.gov.uk/ons/guide-method/measu ring-equality/equality/ethnic-nat-identity-religion/ethnic-group/ index.html\#8. Accessed 2 Mar 2018

Oparil S, Zaman MA, Calhoun DA (2003) Pathogenesis of hypertension. Ann Intern Med 139:761-776

Ormshaw N, Junejo J, Marshall JM (2016) Both endothelium-dependent dilator responses and muscle vasodilator responses to environmental stressors are impaired in young South Asians and may predispose to hypertension. J Hum Hypertens 29:644 PD03

Raji A, Gerhard-Herman MD, Warren M, Silverman SG, Raptopoulos V, Mantzoros CS, Simonson DC (2004) Insulin resistance and vascular dysfunction in nondiabetic Asian Indians. J Clin Endocrinol Metab 89:3965-3972

Rana A, de Souza RJ, Kandasamy S, Lear SA, Anand SS (2014) Cardiovascular risk among South Asians living in Canada: a systematic review and meta-analysis. CMAJ Open 2:E183-191
Ray CJ, Abbas MR, Coney AM, Marshall JM (2002) Interactions of adenosine, prostaglandins and nitric oxide in hypoxia-induced vasodilatation: in vivo and in vitro studies. J Physiol 544:195-209

Schlach MP, Ahlers BA, Parnell MM, Kaye DM (2002) $\beta$ Adrenoceptormediated, nitric-oxide-dependent vasodilatation is abnormal in early hypertension: restoration by L-arginine. J Hypertens 22:1917-1925

Schneider GM, Jacobs DW, Gevirtz RN, O'Connor DT (2003) Cardiovascular haemodynamic response to repeated mental stress in normotensive subjects at genetic risk of hypertension: evidence of enhanced reactivity, blunted adaptation and delayed recovery. J Hum Hypertens 17:829-840

Schwartz CE, Durocher JJ, Carter JR (2011) Neurovascular responses to mental stress in prehypertensive humans. J Appl Physiol 110:76-82

Seddon MD, Chowienczyk PJ, Brett SE, Casadei B, Shah AM (2008) Neuronal nitric oxide synthase regulates basal microvascular tone in humans in vivo. Circulation 117:1991-1996

Tagawa T, Imaizumi T, Endo T, Shiramoto M, Harasawa Y, Takeshita A (1994) Role of nitric oxide in reactive hyperemia in human forearm vessels. Circulation 90:2285-2290

The IPAQ Group (2015) Guidelines for data processing and analysis of the International Physical Activity Questionnaire. http://www. ipaq.ki.se. Accessed 2 Mar 2018

Turpin G, Schaefer F, Boucsein W (1999) Effects of stimulus intensity, risetime, and duration on autonomic and behavioral responding: Implications for the differentiation of orienting, startle, and defense responses. Psychophysiology 36:453-463

Wild SH, Fischbacher C, Brock A, Griffiths C, Bhopal R (2007) Mortality from all causes and circulatory disease by country of birth in England and Wales 2001-2003. J Public Health (Oxf) 29:191-198

Yusuf S, Hawken S, Ounpuu S, Dans T, Avezum A, Lanas F, McQueen M, Budaj A, Pais P, Vargios J, Lisheng L (2004) Effect of potentially modifiable risk factors associated with myocardial infarction in 52 countries (the INTERHEART study): case-control study. Lancet 364:937-952

Zbrozyna AW, Krebbel F (1985) Habituation of the cold pressor response in normo- and hypertensive human subjects. Eur J Appl Physiol 54:136-144

Zbrozyna A, Westwood D (1988) Habituation of vasodilatation in the calf elicited by repeated sensory stimulation in man. Eur J Appl Physiol 58:284-290 